\title{
Modelo para implementar la Norma Técnica de Gestión NTC 6001, Empresas Mypes
}

\section{Model to implement Management Technical Standard NTC 6001, Company Mypes}

págs. 53-70
Grupo de Investigación: Centro de Investigación y Desarrollo Empresarial CINDE
Linea de Investigación: Gestión de calidad y desarrollo de Mipymes
Carlos Gabriel Correa•, Christian David Calderón Calderón •• \& Eliana Carpintero Cárdenas $\cdots$

Recibido: 26 de enero de 2016 Aceptado: 6 de mayo de 2016

\section{Resumen}

El modelo facilita la implementación y certificación en un sistema de gestión NTC 6001 en micro y pequeñas empresas (mypes), equivalente a la NTC ISO 9001, diseñada para todo tamaño de empresa; las mypes al no poder evidenciar dicha implementación. ha venido afectándolas cuando en el proceso de selección de proveedores exigen estar certificadas, las mypes tienen escases en recursos para el proceso de implementarla y lograr la certificación; el ICONTEC, diseñó la norma de gestión NTC 6001 para las mypes, con la que logran formalidad del negocio, normatividad legal, mejoramiento de los procesos administrativos, operativos y de apoyo; su implementación es alcanzable, contribuye en la calidad, seguridad, satisfacción, baja incertidumbre, transformándolas en empresas sostenibles, competitivas con iguales condiciones.

Palabras clave: certificación, formalidad, micros y pequeñas, sistema de gestión, estabilidad.

\begin{abstract}
The model facilitates the implementation and certification management system NTC 6001 micro and small enterprises (MSEs), equivalent to the NTC ISO 9001, designed for all sizes of company; MSEs unable to demonstrate that implementation has been affecting them when in the selection process require suppliers to be certified, MSEs have shortages in resources for the process to implement and achieve certification; ICONTEC, designed the NTC management standard 6001 for MSEs, with which achieved business formality, legal regulations, improving administrative, operational and support processes; its implementation is achievable, contributes to the quality, safety, satisfaction, low uncertainty, transforming them into sustainable, competitive companies with the same conditions.
\end{abstract}

Keywords: certification, formality, micro and small management system, stability

\footnotetext{
- Docente investigador Fundación Universidad de América, carlos.correa@investigadores.uamerica.edu.co

-• Estudiante coinvestigador Fundación Universidad de América. cris_hp25@hotmail.com

... Estudiante coinvestigador Fundación Universidad de América. elsi930@hotmail.com
} 


\section{Introducción}

La implementación del sistema de gestión basada en la norma NTC 6001 mediante el modelo propuesto, se espera cumplir con la exigencia de los clientes que muestran alto grado de incertidumbre en las relaciones comerciales con empresas que no evidencian tener implementado un sistema de gestión, a partir de los requisitos exigidos por la norma en lo que respecta a los procesos administrativos, operativos y de apoyo; a partir de los tres tipos de procesos, se efectuó una lista de chequeo que facilite el diagnóstico para identificar el grado de cumplimiento de los requisitos, para que las empresas micros y pequeñas que representan más del $90 \%$ de las empresas en Colombia (QUINTTERO, 2015), aportan el $38,7 \%$ del PIB, y generan el $57 \%$ de empleo en el país, según estadísticas del DANE (Departamento Administrativo Nacional de Estadística), pero los índices de mortalidad de estas empresas es cada vez más significativa, las causas identificadas en el diagnóstico desarrollado en el trabajo de investigación muestran la falta de formalidad desde el inicio de la empresa, el no cumplimiento de los requisitos de los procesos administrativos, operativos y de apoyo en el desarrollo de la gestión de calidad, relaciones laborales informales, incumplimiento en normatividad técnica y legal; aspectos que facilita su cumplimiento en la implementación de la norma en mención.

Desde su nacimiento en el 2008, la Norma Técnica Colombiana 6001, diseñada por el ICONTEC, se ha implementado y certificado solo 202 empresas en todo el pais; preocupante, la mortalidad se puede controlar si las mypes la implementan desde el inicio del negocio; es una tabla salvadora que organiza desde el principio todo negocio, la pregunta entonces es ¿por qué las micros y pequeñas empresas no implementan dicha norma de gestión?, en el trabajo de investigación se investigó mediante encuesta a los gerentes de las empresas mypes que no han implementado un sistema de gestión y que las hacen vulnerables de no sostenerse en un mercado exigente por el cumplimiento de la norma; las causas o motivos por las cuales las empresas vulnerables identificados en la encuesta desarrollada no implementan la norma, se relacionan en la tabla 1.

\section{Tabla 1.}

Motivos por los cuales la empresa no implementa NTC 6001

\begin{tabular}{lcc}
\hline \multicolumn{1}{c}{ RTA } & Número & Porcentaje \\
\hline No tiene conocimiento de & 97 & $32 \%$ \\
la norma & 11 & $4 \%$ \\
$\begin{array}{l}\text { Falta de interés } \\
\text { Falta de recursos }\end{array}$ & 76 & $25 \%$ \\
económicos & & $18 \%$ \\
$\begin{array}{l}\text { Falta de recursos huma- } \\
\text { nos }\end{array}$ & 54 & $16 \%$ \\
$\begin{array}{l}\text { Exceso de carga adminis- } \\
\text { trativa }\end{array}$ & 49 & $6 \%$ \\
Otro, ¿Cuál? & 19 & $100 \%$ \\
\hline Total & 306 & \\
\hline
\end{tabular}

Nota: Las respuestas por la dirección de empresas mypes, en el desarrollo de la encuesta se observa que las dos causas más representativas corresponden a la falta de conocimiento de la existencia de una norma de gestión que fue diseñada para las empresas mypes, y la falta de recursos para costear y desarrollar el proceso de implementación y luego la correspondiente certificación

Fuente: Trabajo de investigación (Calderón, C \& Carpintero, E (2015)

Oportunidad para divulgar en las empresas mypes, su existencia para su implementación, mediante el modelo que se propone; el número de empresas certificadas por departamento, se muestran a continuación en la tabla 2 , existe un nicho de mercado importante, empezando con Bogotá, D.C, en el que solo 4 empresas se han certificado (Proysolin S.A.S., Suting Ltda., HIf Romero, Leños Express Ltda.), corresponden al $2 \%$ del total de empresas certificadas en el país. 
LIINEA DE INVESTIGACIÓN: GESTIÓN DE CALIDAD Y DESARROLLO DE MIPYMES

Tabla 2.

Número de empresas certificadas por departamento

\begin{tabular}{lcc}
\hline Departamento & $\begin{array}{c}\mathbf{N}^{\circ} \text { de } \\
\text { empresas }\end{array}$ & $\begin{array}{c}\text { Participación } \\
\text { porcentual }\end{array}$ \\
\hline Antioquia & 38 & $18,8 \%$ \\
Arauca & 1 & $0,5 \%$ \\
Bolívar & 9 & $4,5 \%$ \\
Caldas & 20 & $9,9 \%$ \\
Cauca & 3 & $1,5 \%$ \\
Bogotá D.C. & 4 & $2,0 \%$ \\
Guajira & 1 & $0,5 \%$ \\
Huila & 1 & $0,5 \%$ \\
Magdalena & 2 & $1,0 \%$ \\
Quindío & 47 & $23,3 \%$ \\
Risaralda & 10 & $5,0 \%$ \\
Santander & 10 & $5,0 \%$ \\
Valle del cauca & 56 & $27,7 \%$ \\
\hline Total & 202 & $100,0 \%$ \\
\hline
\end{tabular}

Nota: Bogotá como un Distrito capital, donde mayor cantidad de empresas o negocios de tamaño micro y pequeñas se ubican, cuáles han sido las causas por las cuales no se han interesado en la norma NTC 6001?.

Fuente: Trabajo de investigación (Calderón, C \& Carpintero, E (2015)

\section{Método}

Para el desarrollo del trabajo de investigación y el cumplimiento de sus objetivos se aplicó la siguiente metodología:

Método de investigación se basa en el principio de acción participativa (IAP), se caracteriza por ser cualitativo, orientado a una investigación social, direccionada a las ciencias administrativas y socioeconómicas. Para Murcia Florián ${ }^{1}$, el postulado fundamental de la IAP es que el conocimiento de la realidad del objeto es un proceso de transformación a través de la

1 Investigador "Investigar para cambiar" Cooperativa editorial Magisterio, proceso IAP. superación de los conflictos y contradicciones del investigador, del grupo participativo y problema y objeto de estudio.

La IAP fue fundamental en la construcción del modelo, se inició con la selección de las empresas que han implementado la norma, dieron testimonio de la experiencia que tuvieron en su proceso, seguido se analizó las necesidades que presentan las empresas en la implementación de un sistema de gestión, se establecieron los requerimientos de la NTC 6001. Como resultado se diseñó el modelo que facilitará a las empresas, la implementación y así dar solución al problema planteado sobre la necesidad de implementar la norma.

Para el desarrollo de los objetivos se tuvo en cuenta la investigación cualitativa, y datos secundarios, esta información suministrada por entes de administración y seguimiento como el ICONTEC.

\section{Fundamentos teóricos}

El consejo directivo del Instituto Colombiano de Normas Técnicas y Certificación - ICONTEC, ratificó el 26 de marzo de 2008, la NTC 6001: modelo de gestión para micro empresas y pequeñas empresas (Mypes), con el fin de ofrecerle a estas empresas una herramienta que les permita desarrollar una estructura interna sólida que garantice administrar los negocios con estándares de calidad y lograr ser competitivas frente a mercados importantes que les exigen tener implementado y estar certificado en el Sistema de Gestión Calidad y lo especifican en la NTC ISO 9001.

La norma Técnica Colombiana NTC 6001, "Modelo de Gestión para Micro Empresas y Pequeñas Empresas Mypes, establece.requisitos fundamentales para implementar un sistema de gestión en las empresas de cualquier sector económico, tanto de bienes como de servicios, de esta manera permite: 
Demostrar la capacidad de cumplir las exigencias del mercado y los requisitos reglamentarios y legales

Fortalecer su competitividad, teniendo en cuenta el nivel de satisfacción de los clientes y la mejora continua

Orientar al mejoramiento y cumplimiento de requisitos desde su creación:

- Procesos de dirección (planificación y direccionamiento, evaluación de la gestión)

- Procesos operativos (gestión comercial, planificación y desarrollo de productos, gestión de compras, producción de bienes o prestación del servicio)

- Procesos de apoyo (recursos humanos, gestión de información, gestión de recursos físicos)

El total de empresas micros y pequeñas alcanza al $96.4 \%$ y aportan el $52 \%$ de empleo en el país; el nivel de empresas que se registran en la Cámara de Comercio de Bogotá supera los 15000 por año, pero cerca de 13000 terminan con cerrar o liquidar.

Tabla 3.

Tamaños empresa

\begin{tabular}{lcc}
\hline Tipo de empresa & Empleados & $\begin{array}{c}\text { Activos } \\
\text { (SMLMV) }\end{array}$ \\
\hline Microempresa & $1-10$ & Menos de 500 \\
Pequeña empresa & $11-50$ & $501-5000$ \\
\hline
\end{tabular}

Nota: El limite o rango de los activos para el año 2015 calculado de acuerdo con el salario mínimo legal mensual $\$ 644.350$ y para el año 2016 de $\$ 689.455$ corresponde a valores difíciles de contar por parte de las empresas micros o por las empresas pequeñas

Fuente: Ley 590 de 2000 y la ley 905 de 2004

Las empresas mypes, han tenido dificultad, para competir con las empresas medianas y grandes, no pueden acceder al mercado que estas ya han logrado; las empresas medianas y grandes exigen que sus proveedores muestren evidencia de haber implementado y estar certificados en una norma de gestión de calidad; el proceso para lograr dicha implementación y ser certificados, es muy complejo, supera las expectativas de inversión y prefieren efectuar pagos de sus deudas de arriendo, servicios, materia prima que incursionar en el desarrollo de un plan para el cumplimiento de los requisitos y búsqueda de una certificación la cual no es lograble por sus condiciones económicas planteadas.

Las empresas mypes, generalmente inician la gestión empresarial de manera informal en el cumplimiento de normas legales, tributación, legislación laboral, facturación, control de costos, control de producción, documentación, pago oportuno a los proveedores, control de inventarios, planeación de producción, contabilidad y aspectos financieros, entre otros; aspectos que se pueden mejorar y estandarizar en un habitual proceso que se transforme en un sistema de gestión orden y continua mejora, transformándolas en empresas competitivas, no solo a nivel nacional, sino a nivel internacional.

En el informe de coyuntura empresarial en Colombia ${ }^{2}$, entregado por Confecamaras el cual abarca el primer trimestre del año 2014 comparada con el mismo periodo del año 2013, se encuentra:
2 Confecamaras, (2014). Informe de coyuntura Enero - Septiembre 2015,Recuperado 22 de Mayo de 2015 de http://www.confecamaras.org.co/phocadownload/ Informe_de_Coyuntura/Informe_de_Coyuntura_Enero_-Septiembre_2014_v1_2.pdf 
LÍNEA DE INVESTIGACIÓN: GESTIÓN DE CALIDAD Y DESARROLLO DE MIPYMES

Tabla 4.

Empresas creadas Enero - Septiembre 2013/14

\begin{tabular}{lcccc}
\hline & $\mathbf{2 0 1 3}$ & $\mathbf{2 0 1 4}$ & Variación & V \% \\
\hline Sociedades & 49.516 & 54.003 & 4.487 & $9,06 \%$ \\
Personas naturales & 170.248 & 186.247 & 15.999 & $9,40 \%$ \\
\hline Total & $\mathbf{2 1 9 . 7 6 4}$ & $\mathbf{2 4 0 . 2 5 0}$ & $\mathbf{2 0 . 4 8 6}$ & $\mathbf{9 , 3 2 \%}$ \\
\hline
\end{tabular}

Nota: la mayor cantidad de las empresas mypes están en tipo de sociedad de personas naturales, las que corresponden a la tendencia de ser empresas informales, donde no existen generalmente un sistema contable, un sistema de mejoramiento hacia la calidad y buenos procesos

Fuente: RUES - Registro Único Empresarial y Social, 2015

Los anteriores resultados.se ilustran en la siguiente figura como histograma

Figura1.

Ilustración gráfica de la conformación de empresas

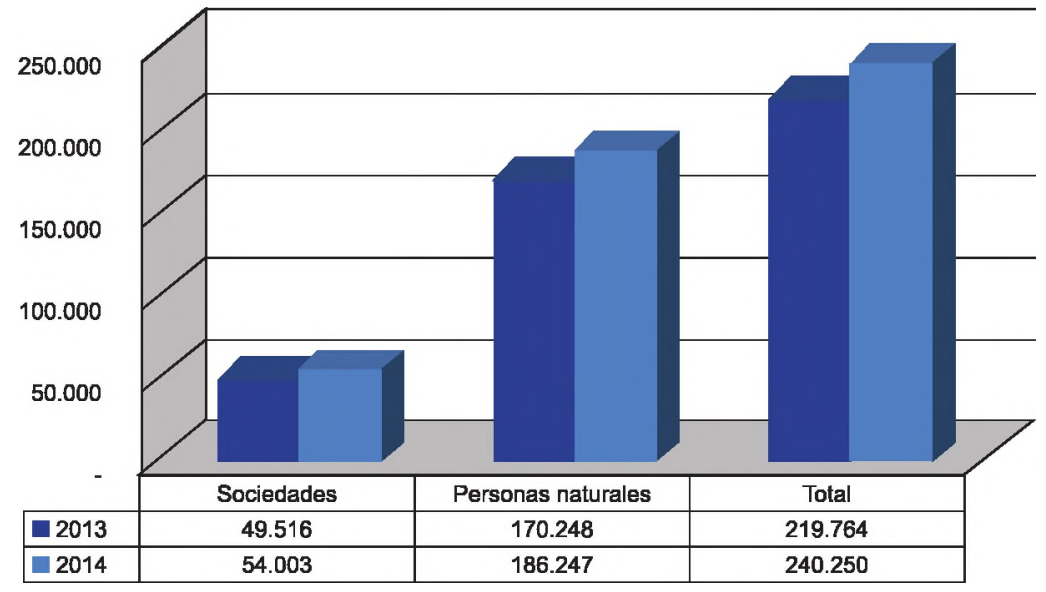

Nota: es notoria la diferencia de conformación de empresas como sociedades, donde se podría esperar que son empresas con mayor esperanza de ser sostenibles en el tiempo, las empresas como personas naturales en los tamaños Mypes, tienden a liquidarse con mayor facilidad

Fuente: Confecamaras Bogotá D.C. 2015

Como desarrollo del modelo, se hace revisión de los procesos de la organización y planteamiento de posibles mejoras referentes a los requisitos de la NTC 6001; se destacan aspectos con una descripción cualitativa a través de un cuadro comparativo con cada uno de los numerales de la norma, posteriormente realizando una ponderación con cada uno de los aspectos se da calificación para un resultado para efectuar un análisis cuantitativo detallado de la situa- ción actual, que refleje aún más la necesidad de la implementación de la NTC $6001 .^{3}$

En la figura 2. Se ilustra la manera de registrar la información que se va tomando en la

3 IRACHETA, José Miguel; PRIDA, Bernardo; ABARCA, Cesar. Metodología práctica para el diseño e implantación de sistemas de la calidad según las normas ISO9000 en pequeñas y medianas empresas. Dirección y Organización, 2010, no 23. 
lista de chequeo a medida que se va evaluando el grado de cumplimiento de cada factor corres- pondiente a cada proceso: administrativo, operativo y de apoyo

Figura 2.

Lista de diagnóstico de cumplimiento norma NTC 6001

\begin{tabular}{|c|c|c|c|c|}
\hline \multicolumn{5}{|l|}{ Lista de verificación NTC 6001:2008 } \\
\hline \multicolumn{2}{|c|}{ N.C- No conforme O.M- Oportunidad de mejora } & \multicolumn{3}{|c|}{ C- Conforme } \\
\hline \multicolumn{5}{|l|}{ Marque con una " $X "$} \\
\hline Requisitos a examinar & N.C & O.M & c. & Observaciones \\
\hline$\uparrow$ & $\uparrow$ & & & $\uparrow$ \\
\hline $\begin{array}{l}\text { Enumeración de } \\
\text { cada uno de los } \\
\text { requisitos exigidos } \\
\text { por la norma }\end{array}$ & & & & $\begin{array}{l}\text { Opiniones generadas } \\
\text { por la empresa a cada } \\
\text { uno de los requisitos } \\
\text { de la norma }\end{array}$ \\
\hline & \multicolumn{2}{|c|}{$\begin{array}{l}\text { Calificación otorgada } \\
\text { según criterio del auditor }\end{array}$} & & \\
\hline
\end{tabular}

Nota: tomando como referente las listas de chequeo diseñadas para hacer revisión como diagnóstico de cumplimento de los requisitos exigidos por la norma, se muestra el método.identificar en cada proceso el grado de cumplimiento de los requisitos que se deben cumplir y que se deben evidenciar ante el ente certificador de la norma

Fuente: Trabajo de investigación (Calderón, C \& Carpintero, E (2015)

El modelo debe ser de fácil interpretación especialmente en lo relativo al criterio que debe tener los facilitadores delo proceso, en lo relativo al grado de cumplimiento de cada factor de- terminado en la lista de diagnóstico, para lo cual se deben seguir los parámetros de calificación de acuerdo a la descripción preestablecida en la tabla 4.

Tabla 4.

Descripción de calificaciones para tabla 5.

\begin{tabular}{ll}
\hline \multicolumn{1}{c}{ Calificación } & \multicolumn{1}{c}{ Descripción del criterio } \\
\hline No conforme & Se otorga cuando la organización no tiene conocimiento o no cumple el numeral \\
Oportunidad de mejora & $\begin{array}{l}\text { Se otorga cuando se detecta el conocimiento sobre el numeral, pero no se cumple } \\
\text { a cabalidad o se presenta una oportunidad de mejora } \\
\text { Conforme }\end{array}$ \\
$\begin{array}{l}\text { Se otorga cuando la organización cumple totalmente con el numeral estudiado de } \\
\text { la norma }\end{array}$ \\
\hline
\end{tabular}

Nota: Con el uso del criterio que se debe seguir se hace una calificación que muestre el grado de cumplimiento de cada factor, en la que se identifica el estado actual o diagnóstico de entrada, para así identificar las oportunidades de mejora que se deben implementar y asi poder justificar la necesidad de su implementación.

Fuente Trabajo de investigación (Calderón, C \& Carpintero, E (2015) 
LÍNEA DE INVESTIGACIÓN: GESTIÓN DE CALIDAD Y DESARROLLO DE MIPYMES

\section{Bases para la Implementación del modelo}

El representante legal de la empresa micro o pequeño toma la decisión de implementar el sistema de gestión de calidad basada en la NTC
6001, para lo cual se inicia con efectuar un diagnóstico general sobre el estado de cumplimiento de los aspectos relevantes, de acuerdo a los factores de evaluación relacionados en la tabla 5.

Tabla 5. Diagnóstico Inicial de cumplimiento con respecto a la NTC - 6001

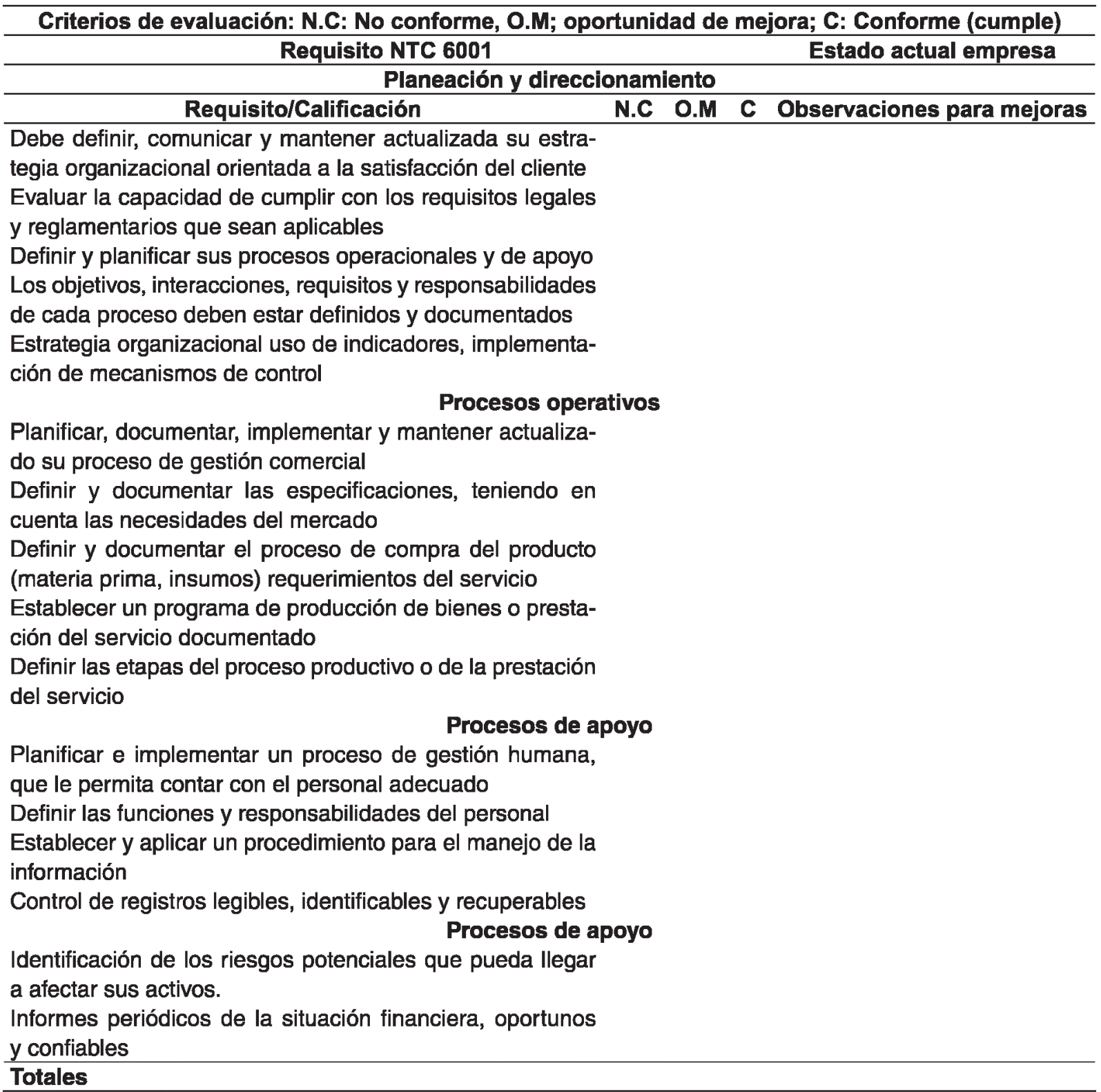

Nota: El resultado ideal corresponde a que todos los parámetros o factores que se evalúan en el listado den como resultado que se encuentran conformes o sea que se cumplen en la totalidad

Fuente: Fuente: Trabajo de investigación (Calderón, C \& Carpintero, E (2015) 
A continuación se ilustra el modelo que se recomienda seguir para implementar el sistema de gestión basado en el proceso de mejoramiento continuo PHV de Edwards Deming; se inicia con la planeación identificando las oportunidades de mejora detectadas en el diagnóstico inicial o llamado de entrada, identificadas las falencias, se hace identificación a partir de las preguntas, que se debe hacer, cómo hacerlas, cuando, en que parte de los procesos, qué se tiene, qué controles se deben implementar, las fechas límites de implementación, asignación de responsables, diseño de indicadores y determinación del estado actual de los indicadores que se propone, esa será la base para hacer continuo seguimiento del avance y de los logros que se van percibiendo, entre ellos el de mejoramiento de la calidad, incremento de ventas por nuevos clientes, satisfacción, relaciones con los proveedores; en la figura 3. Se ilustra el proceso que se recomienda seguir para la implementación del sistema.

\section{Figura 3.}

Proceso de implementación del sistema de gestión NTC 6001

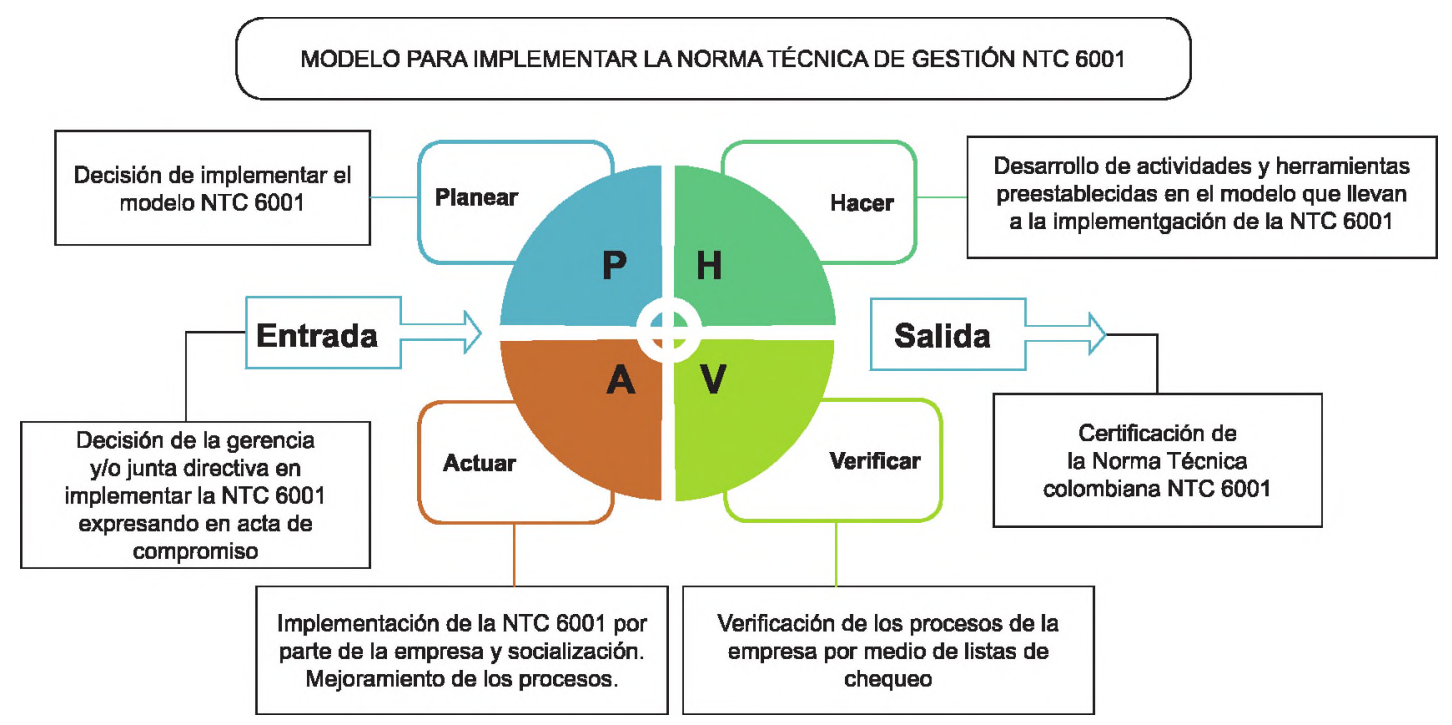

Nota: El proceso de mejoramiento continuo, para el caso de NTC 6001, es una práctica necesaria, que se ha ilustrado en la figura 3

Fuente Trabajo de investigación (Calderón, C \& Carpintero, E (2015)

\section{Proceso de implementación del modelo propuesto}

\subsection{Proceso de Dirección}

Planificación y direccionamiento

- Planificación y direción: Rafael Muñiz Gonzalez

4 Consultor de Márquetin estratégico y operativo
Cualquier empresa que desee tener éxito y busque beneficios debe someterse a un sistema formal de dirección estrategica, es decir, seleccionar y definir perfectamente sus valores dentro de la cadena de valor de la compañía que la hará destacar de la compañía que la hará destacada frente a la competencia.

La empresa debe tener claro su misión y objetivos de manera cuantitativa, se analizan los factores tanto internos como externos que influyen 
LIINEA DE INVESTIGACIÓN: GESTIÓN DE CALIDAD Y DESARROLLO DE MIPYMES

a la empresa con los que se diseñan estrategias, que se ponen en práctica y con indicadores para hacen los controles correspondientes

- Cultura organizacional: Para las empresas micro y empresas pequeñas puede ser fuerte o debil o positiva o negativa, depende del grado de motivación, del grado de apoyo que puede fortalecer los valores, las creecis, hábitos y filosofia en el desarrollo del trabjo; Edgar Shein ${ }^{5}$, dio su concepto claro y práctico al establecer que cada trabajador debe tener independencia, control, por sí mismo, identidad del trabajador en lo que hace, resolución de inconvenientes en los procesos

- Estrategias organizacionales: creación, implementación y evalución de las decisiones dentro de la organización a partir de la misión, la visión y los objetivos de la empresa; para que se logre resultados efectivos, en las empresas mypes, el incluir al personal crea sentido de pertencia por la empresa

- Requisitos legales: la NTC busca superar la tendencia de crear empresas, especialmente las familiares sin la formalidad legal

\subsection{Procesos Operativos}

Planificación del producto y sus procesos de realización

- Planeación y desarrollo del proceso: El primer aspecto para lograr buenos resultados en los diferentes procesos operativos es la planeación, base del proceso de mejora continua PHVA (Método Gerencial Deming, 1950)

Diseño y desarrollo del producto

- Etapas del proceso: es la base para determinar los pasos que se deben cumplir para un buen desarrollo de los productos

5 Psicólogo social, investigador curioso trabajó cerca de Douglas Mc Gregor: autor del concepto "contrato psicológico" no-escrito
- Recursos: qué es necesario respecto a los recursos de materiales, naquinaria, mano de obra y recursos físicos y económicos

- Verificación y validación: se hace verificación de la existencia de los recursos y su validación real

- Objetivo del diseño: si la empresa hace diseño se debe tener claridad del objetivo que cumplirá el producto, que solución busca

\section{Gestión comercial}

- Identificación del mercado objetivo: un mercado objetivo estrechamente definido permite a la empresa enfocar sus esfuerzos; Rene O'Farrel, hace la afirmación:

En negocios, es imposible ser todo para todos los consumidores. Un Toyota, por ejemplo, puede no gustar a todos los compradores de autos sin importar qué tan bueno sea su precio o sus características especiales. Cada persona es diferente. Cada uno tiene sus propias preferencias, sensibilidad al precio, deseo por artículos de lujo y cantidad de ingresos para gastar, haciendo imposible gustar a todo mundo. En su lugar, las empresas concentran sus esfuerzos en una delgada rebanada de población llamada "mercado objetivo".

- Estrategias de ventas: conocido como el arte de proyectar y dirigir las operaciones militares, especialmente las de guerra, llevado a las empresas, es gran debilidad de las empresas micros y de las empresas pequeñas, generalmente se inicia la empresa, esperando a los clientes se acerquen al negocio, la estrategia en las ventas se debe implementar; Emigdio Rafael Contreras ${ }^{6}$ afirma en su articulo de investigación

Definir el alcance, lo que se quiere ser y establecer, y cuál es la razón de ser de la empresa son algunos de esos aspectos im-

6 Autor artículo: El concepto de estrategia como fundamento de planeación estratégica, julio de 2013, revista Pensamiento y gestión, Nº 35 ISSN 1657-6276, pág. 154 
portantes que van a permitir plantear las estrategias necesarias para cumplir con lo que se espera.

- Verificación satisfacción del cliente: falencia o debilidad de estas empresas, las micro y pequeñas, debe implementar la práctica retroalimentación de la satisfacción del cliente en las operaciones de entrega de productos o de servicios.

\section{Gestión de compras}

- Especificación del producto o material: de acuerdo con las características del producto originada por el cliente, se especifican las necesidades de material tanto en lo que respecta a las cantidas que se necesitan por cada orden de trabajo, de esta manera se hace programación de suministros.

- Selección proveedor: cada proveedor debe cumplir con condiciones que son determinadas como factores de decisión: representación, servicio posventa, confianza, capacidad, condiciones de pago, como otro factor el precio, factor que no debe ser de prioridad en la decisión de la compra.

- Contrato de compra: de acuerdo a la selección de proveedor, cada periodo se debe hacer un contrato de las condiciones de compra, para no tener sorpresas durante el recorrido del tiempo

- Recepción tecnica: un proceso de gran importancia es la recepción técnica de lo que se compra, lo debe hacer el almacenista con conocimiento del técnico del material que recibe y el conocimiento de las cantidades que debe recibir; el almacenista debe avisar de manera inmediata sobre la recepción del material

\subsection{Procesos de apoyo}

Descripción de cargos y políticas. Describir de manera formal el trabajo que debe des- empeñar el personal tanto administrativo como operativa o de producción

- Política de contrato: la alta dirección debe establecer directrices para que tanto el empleador como el empleado no se encuentre con sorpresas y de esta manera se establecen condiciones estables para unas buenas relaciones

- Política de bienestar: la empresa promuebe y ejecuta acciones tendientes a crear un ambiente de bienestar, que su tiempo de trabajo sea agradable.

- Reglamento interno de trabajo: es obligación contar con un reglamento interno de trabajo, en que haya claridad sobre aspectos como horarios, horas extras, prohibiciones.

- Plan de capacitación: no basta con la experiencia del trabajador, un mundo en constante evolución exige que toda organización debe tener prioridad respecto a la capacitación de su personal

- Evaluación de desempeño: el desempeño de cada trabajador debe evaluarse por ciclos comparando los resultados obtenidos con los resultados esperados

- Seguridad en el trabajo: todo trabajador al ser vinculado generalmente cumple ademas de la experiencia, conocimiento, habilidad y demás factores, un estado de bienestar físico mental y social o sea cumple con la salud para desempeñarse; la empresa tiene la obligación de mantenerla, mejorarla y preservarsela ofreciendo condiciones de trabajo seguros y saludables

- Gestión de la información: la empresa debe establecer un sistema que facilite controlar los documentos y registros que muestren la formalidad de la empresa, así como orden en la documentación contable, registro actualizado ante la Cámara de Comercio, aportes parafiscales, aportes al sistema general de riesgos 
LÍNEA DE INVESTIGACIÓN: GESTIÓN DE CALIDAD Y DESARROLLO DE MIPYMES

\subsection{Gestión Financiera}

- Estados financieros: como resultado de la contabilidad se deben hacer informes para conocer la situación contable de la empresa corespondientes a: estado de liquidez, rentabilidad, estado de cartera

- Análisis de resultados financieros: todo resultado de los estados financieros se deben analizar para conocer el estado real de la empresa, se debe transformar en un habito administrativo en lo posible en periodos cortos.

\section{Sistema de auditoria interna basado en la NTC 6001}

Como instrumento de diagnóstico del estado final, se efectúa evaluación de cumplimiento para cada uno de los procesos identificados en la norma NTC 6001, para facilitar su gestión se relacionan en las tablas $6,7,8,9,10,11,12,13$, 14,15, y 16 el cumplimiento para determinar y solicitar al ente certificador ICONTEC, la evaluación de auditoria externa.

Cada uno de los numerales se evalúa de acuerdo a los siguientes valores: 0 : En nada cumple el requisito evaluado; 1 : Cumple parcialmente; 2: Cumple aceptablemente; 3: Cumple plenamente.

Tabla 6.

Diagnóstico Planificación y Direccionamiento

\begin{tabular}{lccccc}
\hline \multirow{2}{*}{ Numeral } & Procesos de dirección & \multicolumn{3}{c}{ Cumplimiento } \\
\cline { 2 - 5 } & Planificación y direccionamiento & 0 & 1 & 3 \\
\hline
\end{tabular}

3.1.1.1 La dirección define, comunica y mantiene actualizada su estrategia organizacional orientada a la satisfacción del cliente y a la mejora continua).

3.1.1.2 La dirección idéntica, implementa y mantiene uno o varios procedimientos para evaluar la capacidad de cumplir con los requisitos legales y reglamentarios que sean aplicables y otros requisitos, dependiendo del sector en el cual realiza sus actividades o el mercado al cual dirige sus bienes o servicios o ambos.

3.1.1.3. La dirección define y planifica sus procesos, considerando que estos son de dirección, operacionales y de apoyo.

3.1.1.4 Los objetivos, las interacciones, los requisitos y las responsabilidades de cada proceso están definidos y documentados

3.1.1.5 Los procesos de la organización son suficientes y coherentes con la estrategia organizacional que se determinó 
Tabla 7.

Diagnostico Evaluación de la Gestión

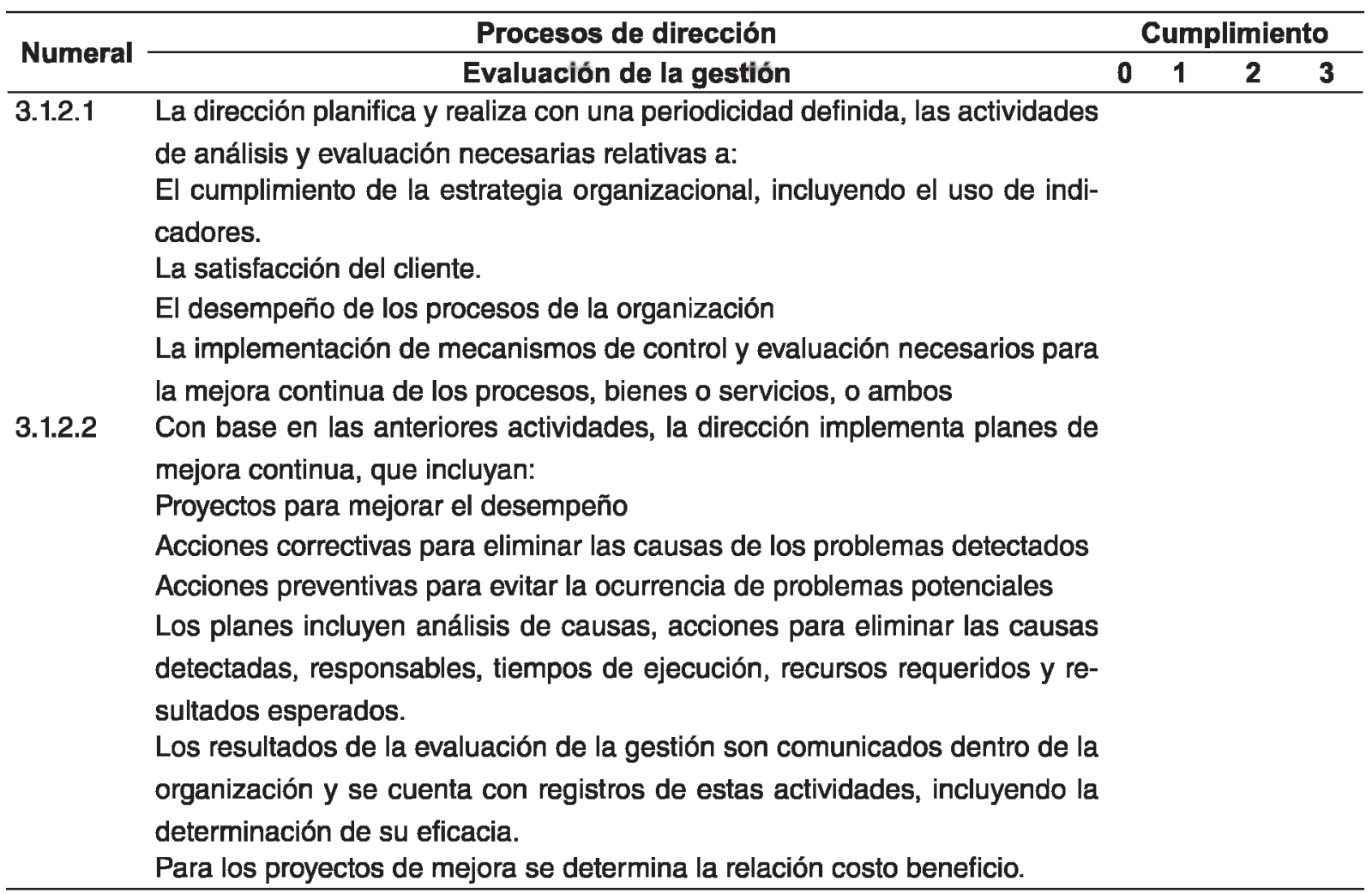

Tabla 8.

Diagnóstico Gestión Comercial

\begin{tabular}{|c|c|c|c|c|c|}
\hline \multirow{2}{*}{ Numeral } & \multirow{2}{*}{$\begin{array}{l}\text { Procesos operativos } \\
\text { Gestión comercial }\end{array}$} & \multicolumn{4}{|c|}{ Cumplimiento } \\
\hline & & 0 & 1 & 2 & 3 \\
\hline
\end{tabular}

La empresa planifica, documenta y mantiene actualizado su proceso de gestión comercial que incluye las siguientes actividades como mínimo:

- Identifica el mercado objetivo.

- Investiga las necesidades y expectativas del mercado objetivo.

- Analiza la competencia y determina las ventajas competitivas.

- Define las políticas y estrategias de ventas.

3.2.1 - Establece el sistema de comercialización de producto.

- Formaliza los acuerdos y compromisos con los clientes, con respecto a los productos, condiciones de entrega y condiciones comerciales.

- Realiza las actividades de venta y distribución del producto o servicio o ambos de acuerdo con lo pactado con el cliente.

- Realiza seguimiento y servicio posterior a la entrega, incluyendo la atención de quejas y reclamos.

- Evalúa la satisfacción de las necesidades del cliente. 
LÍNEA DE INVESTIGACIÓN: GESTIÓN DE CALIDAD Y DESARROLLO DE MIPYMES

Tabla 9.

Diagnóstico Planificación de Productos

\begin{tabular}{|c|c|c|c|c|c|}
\hline \multirow{2}{*}{ Numeral } & \multirow{2}{*}{$\begin{array}{c}\text { Procesos operativos } \\
\text { Planificación de productos }\end{array}$} & \multicolumn{4}{|c|}{ Cumplimiento } \\
\hline & & 0 & 1 & 2 & 3 \\
\hline 3.2.2.1 & $\begin{array}{l}\text { La empresa define y documenta las especificaciones, teniendo en cuenta las } \\
\text { necesidades del mercado, los requisitos técnicos y las necesidades del cliente, } \\
\text { aspectos legales y reglamentarios de los productos por suministrar. }\end{array}$ & & & & \\
\hline
\end{tabular}

Tabla 10.

Diagnóstico Planificación y Desarrollo de Procesos

\begin{tabular}{llccc}
\hline \multirow{2}{*}{ Numeral } & \multicolumn{1}{c}{ Procesos operativos } & Cumplimiento \\
\cline { 2 - 5 } & \multicolumn{1}{c}{ Planificación y desarrollo de procesos } & $\mathbf{0}$ & $\mathbf{2}$ & $\mathbf{3}$ \\
\hline 3.2.2.2 & $\begin{array}{l}\text { Planifica y documenta el proceso de realización del producto en función de } \\
\text { sus especificaciones, necesidades de los procesos y de las proyecciones de } \\
\text { innovación }\end{array}$ & & \\
\hline
\end{tabular}

Tabla 11.

Diagnóstico Diseño y Desarrollo de Productos

\begin{tabular}{|c|c|c|c|c|}
\hline \multirow{2}{*}{ Numeral } & \multirow{2}{*}{$\begin{array}{c}\text { Procesos operativos } \\
\text { Diseño y desarrollo de productos } \\
\end{array}$} & \multicolumn{3}{|c|}{ Cumplimiento } \\
\hline & & 01 & 2 & 3 \\
\hline 3.2 .3 & $\begin{array}{l}\text { Define o modifica las características de sus productos, según las exigencias de } \\
\text { los clientes, del mercado y los proyectos de innovación, establece, documenta y } \\
\text { ejecuta un plan de diseño que incluye: objetivo del diseño, etapas, cronograma, } \\
\text { responsabilidades, recursos y resultados esperados }\end{array}$ & & & \\
\hline
\end{tabular}

Tabla 12.

Diagnóstico Gestión de Compras

\begin{tabular}{|c|c|c|c|c|c|}
\hline \multirow{2}{*}{ Numeral } & \multirow{2}{*}{$\begin{array}{l}\text { Procesos operativos } \\
\text { Gestión de compras } \\
\end{array}$} & \multicolumn{4}{|c|}{ Cumplimiento } \\
\hline & & 0 & 1 & 2 & 3 \\
\hline 3.2.4 & $\begin{array}{l}\text { - Define y documenta el proceso de compra del producto (materias primas, } \\
\text { insumos, productos terminados, entre otros) o requerimientos del servicio. } \\
\text { - Define y documenta las especificaciones del producto que se va a comprar, } \\
\text { teniendo en cuenta si aplica, las especificaciones definidas en normas na- } \\
\text { cionales o internacionales. } \\
\text { - Define y documenta previamente las necesidades o requerimientos de compra } \\
\text { de materias primas, insumos, productos terminados, de bienes o servicios. } \\
\text { - Define y documenta los criterios de selección de los proveedores y registro } \\
\text { de su cumplimiento. } \\
\text { Define y mantiene las condiciones adecuadas de almacenamiento para la } \\
\text { preservación y conservación de los productos adquiridos. } \\
\text { Define y documenta el proceso de compra del producto (materias primas, } \\
\text { insumos, productos terminados, entre otros) o requerimientos del servicio. } \\
\text { Define y documenta las especificaciones del producto que se va a comprar, } \\
\text { teniendo en cuenta si aplica, las especificaciones definidas en normas na- } \\
\text { cionales o internacionales. } \\
\text { Define y mantiene las condiciones adecuadas de almacenamiento para la } \\
\text { preservación y conservación de los productos adquiridos. }\end{array}$ & & & & \\
\hline & & & & & \\
\hline
\end{tabular}


Tabla 13.

Diagnóstico Producción de bienes o prestación del servicio

\begin{tabular}{|c|c|c|c|c|c|}
\hline \multirow{2}{*}{ Numeral } & \multirow{2}{*}{$\begin{array}{c}\text { Procesos operativos } \\
\text { Producción de bienes o prestación del servicio }\end{array}$} & \multicolumn{4}{|c|}{ Cumplimiento } \\
\hline & & $\mathbf{0}$ & 1 & 2 & 3 \\
\hline
\end{tabular}

3.2.5 La empresa establece un programa de producción de bienes o prestación del servicio, documentado que incluya:

Tipos de producto por generar:

Define y asigna la capacidad requerida:

Asigna los recursos (incluyendo materias primas, insumos, personal, información entre otros):

Define las etapas del proceso productivo o de la prestación del servicio:

Fabrica el bien o presta el servicio bajo las condiciones especificadas.

Identifica el producto, si es aplicable, en cada una de las etapas de producción o prestación del servicio:

Establece y aplica los métodos del control de proceso:

Realiza la validación del proceso productivo, del producto o prestación del servicio de acuerdo con lo planificado

Establece y aplica los métodos de verificación, tales como ensayos o inspecciones, con el fin de evaluar la conformidad del producto con los requisitos especificados,

\section{Producción de bienes o prestación del servicio}

Presenta y entrega el producto según los requisitos especificados:

Define una metodología para el control de los inventarios de materia prima, producto en proceso y producto terminado:

Define y mantiene las especificaciones adecuadas de almacenamiento para la preservación y conservación de los productos:

\section{Tabla 14.}

Diagnóstico Gestión de la Información

\begin{tabular}{|c|c|c|c|c|c|}
\hline \multirow{2}{*}{ Numeral } & \multirow{2}{*}{$\begin{array}{c}\text { Procesos de apoyo } \\
\text { Gestión de la información }\end{array}$} & \multicolumn{4}{|c|}{ Cumplimiento } \\
\hline & & $\mathbf{0}$ & 1 & 2 & 3 \\
\hline \multirow[t]{5}{*}{3.3 .2} & $\begin{array}{l}\text { La empresa establece y aplica un procedimiento para el manejo de la informa- } \\
\text { ción que permite: }\end{array}$ & & & & \\
\hline & Tener acceso a la información confiable y oportuna. & & & & \\
\hline & Analizar e interpretar adecuadamente la información. & & & & \\
\hline & Proteger la información relevante para las actividades de la empresa. & & & & \\
\hline & $\begin{array}{l}\text { Esta información sirve de base para la evaluación de la gestión según su rele- } \\
\text { vancia. }\end{array}$ & & & & \\
\hline
\end{tabular}


LÍNEA DE INVESTIGACIÓN: GESTIÓN DE CALIDAD Y DESARROLLO DE MIPYMES

\begin{tabular}{|c|c|c|c|c|c|}
\hline \multirow{2}{*}{ Numeral } & \multirow{2}{*}{$\begin{array}{c}\text { Procesos de apoyo } \\
\text { Gestión de la información }\end{array}$} & \multicolumn{4}{|c|}{ Cumplimiento } \\
\hline & & 0 & 1 & 2 & 3 \\
\hline 3.3.2.1 & $\begin{array}{l}\text { Los documentos requeridos y los que la empresa determina o la ley exija como } \\
\text { necesarios están formalmente aprobados por la autoridad que se define para tal } \\
\text { fin, están vigentes y disponibles para su consulta y aplicación por las personas } \\
\text { que lo requieren, también se evita el uso de documentos obsoletos }\end{array}$ & & & & \\
\hline 3.3.2.2 & $\begin{array}{l}\text { Los registros que evidencian la operación conforme y eficaz del sistema de } \\
\text { gestión frente a los requisitos de esta norma, y los que la empresa determina } \\
\text { como necesarios, están legibles, identificables y recuperables. Se mantienen } \\
\text { almacenados en forma segura y ordenada, durante el tiempo de conservación } \\
\text { que define la empresa o define la ley. }\end{array}$ & & & & \\
\hline
\end{tabular}

Tabla 15.

Diagnóstico Gestión Financiera

\begin{tabular}{|c|c|c|c|c|c|}
\hline \multirow{2}{*}{ Numeral } & \multirow{2}{*}{$\begin{array}{l}\text { Procesos de apoyo } \\
\text { Gestión financiera }\end{array}$} & \multicolumn{4}{|c|}{ Cumplimiento } \\
\hline & & 0 & 1 & 2 & 3 \\
\hline 3.3.3 & $\begin{array}{l}\text { La empresa mantiene actualizada la información relacionada con } \\
\text { - La identificación de los riesgos potenciales que pueden llegar a afectar los } \\
\text { activos y establecidas las acciones preventivas necesarias para salvaguar- } \\
\text { darlos } \\
\text { - Los costos de los bienes o servicios y mantener registros de los cálculos } \\
\text { de éstos } \\
\text { - El nivel mínimo de operación (punto de equilibrio) para no tener pérdidas } \\
\text { - Los informes periódicos de la situación financiera, oportunos y confiables }\end{array}$ & & & & \\
\hline
\end{tabular}

Tabla 16.

Diagnóstico Gestión de Recursos Físicos

\begin{tabular}{cccccc}
\hline \multirow{2}{*}{ Numeral } & \multicolumn{2}{c}{ Procesos de apoyo } & \multicolumn{3}{c}{ Cumplimiento } \\
\cline { 2 - 5 } & Gestión de recursos físicos (maquinaria, instalaciones y equipos) & 0 & 1 & 2 & 3 \\
\hline
\end{tabular}

\subsubsection{La empresa}

- Dispone de la infraestructura, maquinaria, equipos y condiciones necesarias para cumplir los requisitos de los procesos de los productos

- Determina y planifica el mantenimiento de la infraestructura y de los equipos que le permiten su funcionamiento adecuado

- Tiene verificados y calibrados los equipos de medición y los correspondientes registros

Nota: como resultado se elabora gráfica de radar que concluye los resultados de la auditoría de cada uno de los procesos: administrativos, operativos y de apoyo

Fuente Trabajo de investigación (Calderón, C \& Carpintero, E (2015) 
Ejemplo: Procesos operativos: resultado real de diagnóstico y los valores de cumplimiento del
$100 \%$, ver tabla 17 , se relaciona los resultados del diagnóstico y los valores esperados

Tabla 17. Ejemplo de valores reales de resultados de procesos operativos

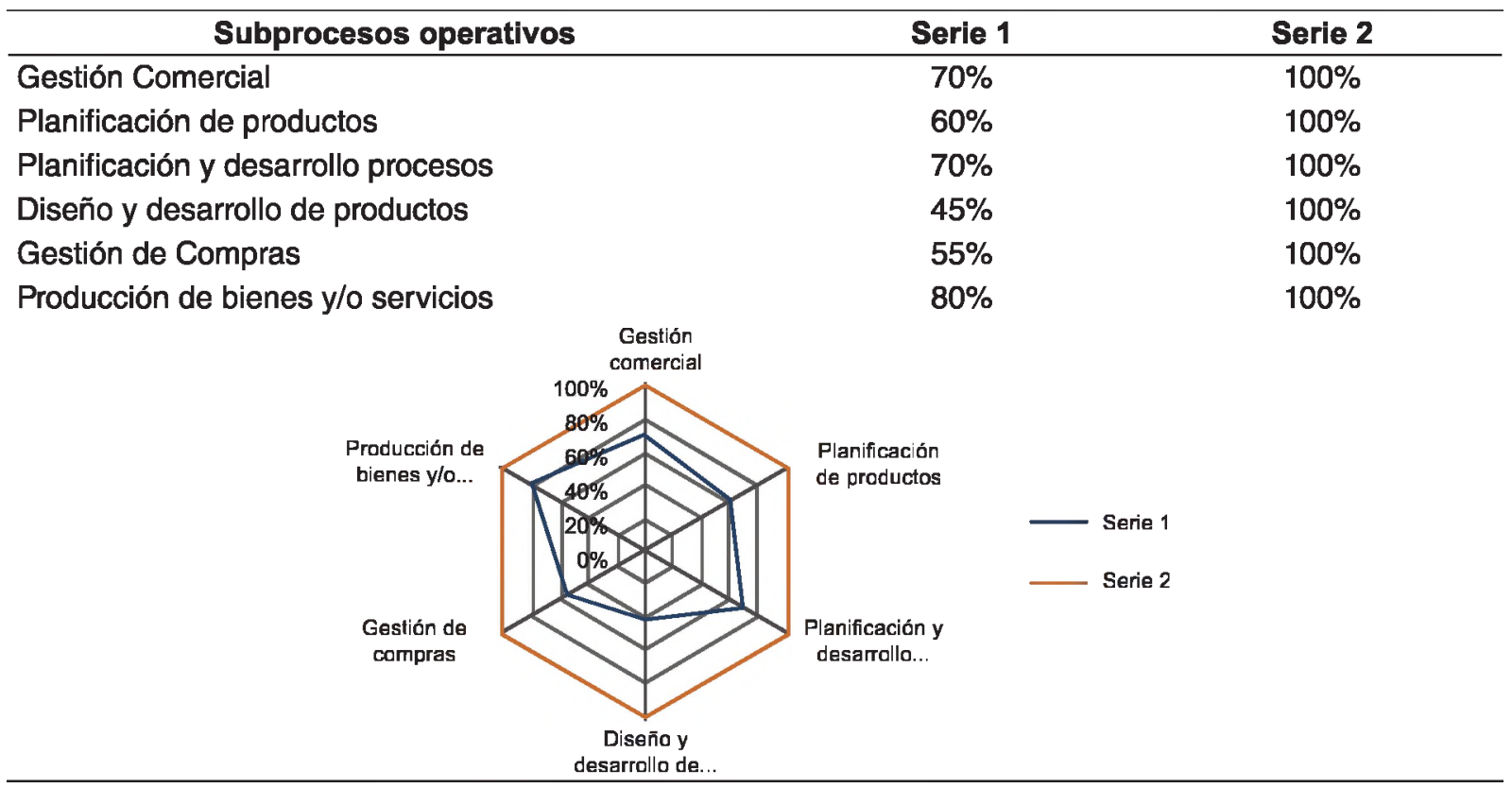

\section{Comentarios}

La razón por la cual se realizó el modelo para implementar la NTC 6001, diseñada por Icontec, se fundamentó en la necesidad que tienen las empresas mypes de implementar un sistema de gestión de calidad equivalente a la norma de gestión de calidad NTC ISO 9001, implementación que exigen las empresas medianas y grandes a los proveedores, para poder participar como suministradores de productos 0 servicios.

En Colombia la mayor concentración de empresas está en las micros y pequeñas, son las que mayor cantidad inician, pero no muestran estabilidad, crecimiento y competitividad, una de las mayores causas son el alto grado de informalidad en su administración, en sus controles y seguimientos de producción y control de costos, informalidad en la contabilidad y no existencia de estados financieros para la toma de decisiones, no se hace seguimiento a la sa- tisfacción de los clientes, no muestran evidencia de hacer planeación, no cuentan con un sistema de información que a partir de indicadores les facilite la toma de decisiones.

Las empresas mypes en su mayoría, no tienen conocimiento de la existencia de esta norma y por motivo de presentarse oportunidad de cotizar y participar en licitaciones toman la decisión apresurada de dedicarse a estudiar los requisitos de la norma de gestión de calidad bajo la norma NTC ISO 9001, cuando ya se encuentran en el proceso, algunas abandonan dicho objetivo; si conocieran la norma equivalente, y apoyada por el modelo que se propone en este artículo, podrían alcanzarlo y al implementarlo, la empresa alcanzaria un mejor estatus, de competitividad, estabilidad, crecimiento que redunda en no ser otra empresa que termina cerrando como otras, que terminan saliendo del mercado 
LIINEA DE INVESTIGACIÓN: GESTIÓN DE CALIDAD Y DESARROLLO DE MIPYMES

La creación y la continua cancelación de empresas mypes, es un indicador preocupante que motiva a analizar la necesidad de implementar el sistema de Gestión NTC 6001, norma diseñada para ser implementada en estos tamaños de empresas

La exigencia que hacen las empresas a sus proveedores de evidenciar haber implementado un sistema de gestión de calidad, es un obstáculo que pone en desventaja a las empresas Mypes, que han tenido la experiencia de no tener los recursos y capacidad para implementar la norma más conocida la NTC ISO 9001.

El objetivo primordial de la norma NTC 6001, es el de mejorar la organización interna sólida y altos estándares de calidad, aportar al objetivo de lograr que las empresas sean competitivas, sostenibles y de continuo crecimiento.

Del total de empresas micros y pequeñas de Bogotá D.C., solo 4 han implementado y se han certificado en la NTC 6001

La implementación de la norma NTC 6001, hace que las empresas desde el inicio se for- malicen desde el conocimiento del mercado, las fortalezas y las debilidades y de esta manera establecer estrategias a implementar en cada uno de los procesos tanto administrativos, operativos y de apoyo.

\section{Recomendaciones}

Divulgar la existencia de la norma NTC 6001 , a las empresas Mypes ( $96 \%$ del total de empresa colombianas), conozcan esta alternativa para cumplir con la exigencia de las empresas de haber implementado y estar certificado en un sistema de gestión de calidad.

Desde inicio de la empresa, debe conocer la norma, y cumplir con los requisitos, que al empezar el negocio, lo organiza, lo hace formal al implementar el cumplimiento de aspectos incluyendo la normatividad legal, propia y fundamental del tipo de empresa o negocio

En la renovación del registro mercantil que debe hacer toda empresa cada año (antes del 31 de marzo)

\section{Referencias}

Artículo "El concepto de estrategia como fundamento de planeación estratégica". Pensamiento \& gestión, 35. Universidad del Norte, N 35 ISSN 1657-6276. Emigdio Rafael Contreras Sierra :emigdio.contreras@cecar.edu.co

NTC - ISO 9000, Norma Técnica Colombiana, Sistemas de gestión de la calidad. Fundamentos y vocabulario, 2005.

NTC - ISO 9001, Norma Técnica Colombiana, Sistemas de gestión de la calidad. Requisitos, borrador 2015.

NTC - ISO 9004, Norma Técnica Colombiana, Gestión para el éxito sostenido de una organización. Enfoque de gestión de la calidad, 2010.

NTC - 6001, Norma Técnica Colombiana, Modelo de gestión para micro empresas y pequeñas em-presas (MYPES), 2008. 
Confecamaras, (2014). Informe de coyuntura Enero - Septiembre 2015, Recuperado 22 de Mayo de 2015 de http://www.confecamaras.org.co/phocadownload/Informe_de_Coyuntura/Informe_de_Coyuntura_Enero_-_Septiembre_2014_v1_2.pdf

Cultura organizacional desde la teoría de Edgar Schein: Estudio fenomenológico http://pyme.lavoztx.com/identificacin-del-mercado-objetivo-12241.html

Escrito por Renee O'Farrell, Demand Media | Traducido por lliana Koste.

Francés, A. (2006). Estrategia y planes para la empresa con el cuadro de mando integral. Pearson educación de México S.A. de C.V.

Quintero Arteaga, Marcela, et al. Sistema de Gestión de la Calidad en las PYMES Colombianas (2015) http://pyme.lavoztx.com/identificacin-del-mercado-objetivo-12241.html

Estadísticas de la microempresa en Colombia propais.org.co/biblioteca/observtorio/estadística-sobre-las-microempresas

Método para implementar la norma Técnica de Gestión NTC 6001, en la empresa Andes Ingeniería de Proyectos Christian David Calderón Calderón \&Eliana Carpintero Cárdenas 2015. 